\title{
A Transformer Condition Assessment System Based on Data Warehouse and Data Mining
}

\author{
Xueyu $\mathrm{Li}^{1}$, Lizeng $\mathrm{Wu}^{2}$, Jinsha Yuan ${ }^{1}$, and Yinghui Kong ${ }^{1}$ \\ ${ }^{1}$ Department of Electronics and Communication, North China Electric Power University \\ No.204, Qingnian Road, Baoding, 071003, China \\ ${ }^{2}$ Beijing No.2 Co-generation Plant No. 52, Lian Hua Chi Dong Lu, Beijing, China \\ lxueyu@gmail.com \\ lizengwulyahoo.com.cn \\ yuanjinsha@sohu.com \\ kongyhesina.com
}

\begin{abstract}
A framework of transformer condition assessment system is proposed in this paper. In this system, we use a data warehouse, a multi-agent system and data mining techniques respectively to collect transformers' testing data, design the framework of the software, and evaluate transformers' conditions. The proposed system prototype had been tested with realistic transformers with reliable performance. The present framework is open and flexible, therefore the objective system is easy to be maintained and developed further.
\end{abstract}

Keywords: Transformer condition assessment, data mining, multi-agent system.

\section{Introduction}

Power utilities are under continuous pressure to reduce maintenance expenditures while maintaining a high level of component reliability. As a result, condition based maintenance (CBM) has been developed to cut down the maintenance cost and increase the level of system and component reliability. An open substation main equipment state monitoring system framework, which used data warehouse technologies to collect all kinds of data and used data mining and Open Architecture Agent technologies to set up an open architecture, is proposed in this paper.

\section{A New Transformer Condition Assessment System}

The new overall transformer condition assessment system (TCAS) is a hybrid system that is composed of a data collection subsystem and a condition analysis subsystem. The former collects transformers' on-site monitoring data, off-line present and historical testing data, nameplate parameters, and historical operating records into a data warehouse. The later uses the data in the data warehouse to evaluate the conditions of all concerned transformers in an electric utility. 


\subsection{Data Collection Subsystem}

Data warehouse is used in the data collection subsystem for data collection. In addition, an OLAP tool is included in the subsystem to enable easy and efficient data analysis tasks by the users.

\subsection{Condition Analysis Subsystem}

\subsubsection{Architecture of the Transformer Condition Analysis Subsystem}

The transformer condition analysis subsystem includes the following seven application agents:

$$
\begin{gathered}
\mathrm{AA}=\left\{\mathrm{AA}_{\text {Threshold_Alarm }}, \quad \mathrm{AA}_{\text {Trend_Analysis, }}, \quad \mathrm{AA}_{\text {EventTree_Analysis, }}, \quad \mathrm{AA}_{\text {Cluster_Analysis, }}\right. \text {, } \\
\left.\mathrm{AA}_{\text {Classification_Analysiss }}, \mathrm{AA}_{\text {Forecasting }}, \mathrm{AA}_{\text {Condition_Assessment }}\right\}
\end{gathered}
$$

The above application agents work together to evaluate transformers' conditions. The knowledge of these agents can be described as follows:

$\mathrm{KAA}_{\text {Threshold_Alarm }}=\{$ dissolved gases concentrations, gases production speeds, electric testing data, ratio limit, speed limit, electric data limits $\}$

$\mathrm{KAA}_{\text {Trend_Analysis }}=\{$ dissolved gases concentrations, gases production speeds, electric testing data $\}$

$\mathrm{KAA}_{\text {Event Tree_Analysis }}=\{$ entrance circuit short, continuous high temperature, sustained overload, environmental abnormity, fault analysis $\}$

$\mathrm{KAA}_{\text {Cluster_Analysis }}=\{$ dissolved gases concentrations, dissolved gases relative ratios, electric testing data, grey relation clustering algorithm $\}$

$\mathrm{KAA}_{\text {Classification_Analysis }}=\{$ dissolved gases relative ratios, electric testing data, Bayesian network classifier $\}$

$\mathrm{KAA}_{\text {Forecasting }}=\{$ dissolved gases concentrations, dissolved gases relative ratios, electric testing data, grey prediction algorithm $\}$

$\mathrm{KAA}_{\text {Condition_Assessment }}=\{$ threshold analysis results, trend analysis results, event tree analysis results, cluster analysis results, classification results, forecasting results, Bayesian network model $\}$

\subsubsection{Application Agents}

2.2.2.1 Threshold Alarm Agent. Standard value analysis agent is used to compare the actual tested value derived from testing data, on-line monitoring data and other data with standard values of transformer condition.

2.2.2.2 Trend Analysis Agent. The quality indices of a transformer usually change with time extension. If they change slowly and they are within the ranges of their standard values, the transformer is in normal condition. If they change sharply, usually a hidden failure or defect occurs in the transformer even though they are within the ranges of their standard values.

2.2.2.3 Event Tree Analysis Agent. Event tree analysis and fault tree analysis are two main methods to do the transformer fault analysis. When an event, such as external short circuit, continuous high environmental temperature, sustained overload, happen, the event tree analysis agent can find it from recorded data and then do condition assessment. 
2.2.2.4 Cluster Analysis Agent. Cluster analysis agent is responsible for finding the testing data's distribution patterns and relations of attributes. Cluster analysis agent responds to the request of the facilitator agent to make the cluster analysis. The grey correlation method is used to extract the failure types.

2.2.2.5 Classification Analysis Agent. The naïve Bayesian classifier learns from training data the conditional probability of each attribute $\mathrm{A}_{\mathrm{i}}$ given the class label $\mathrm{C}$. Classification is then done by applying Bayes rule to compute the probability of $\mathrm{C}$ given the particular instance of $A_{1}, \ldots, A_{n}$, and then predicting the class with the highest posterior probability.

2.2.2.6 Forecasting Agent. Forecasting agent responds to the request of the facilitator agent, which provides the agent community with a number of services for routing and delegating tasks and information among agents, to make the transformer failure forecast. In grey model $\operatorname{GM}(1,1)$, stochastic variables are seen as Grey Variables, irregular original data are cumulated to be regular series, and then, a differential equation model is formed and settled. When the grey theory is used to predict transformers' dissolved gases values, the posteriori error of each gas can be about $5 \%$ or less than $5 \%$ [11].

2.2.2.7 Transformer Condition Assessment Agent. The threshold analysis, trend analysis, event tree analysis, cluster analysis, classification analysis, and parameter forecasting are comprehensively analyzed through using the Bayesian network. The transformer condition is divided into five states, excellent, better, normal, worse and fault. Transformer condition node has seven parent nodes, which are all middle nodes just like threshold analysis result node.

\section{Applications}

We are developing a transformer condition assessment system for an electric utility. The initial results expressed in table 1 are suitable to actual transformers' conditions.

Table 1. Initial results

\begin{tabular}{ccccccccc}
\hline \multirow{2}{*}{$\begin{array}{c}\text { Voltage grade } \\
(\mathbf{k V})\end{array}$} & $\begin{array}{c}\text { Capacity } \\
(\mathbf{k V A})\end{array}$ & Count of Tr. & \multicolumn{4}{c}{$\begin{array}{c}\text { Transformers' } \\
\text { Conditions }\end{array}$} \\
\cline { 4 - 8 } & & & $\mathbf{E}$ & $\mathbf{B}$ & $\mathbf{N}$ & $\mathbf{W}$ & $\mathbf{F}$ \\
\hline 110 & 31500 & 28 & 11 & 14 & 1 & 1 & 1 \\
110 & 20000 & 7 & 6 & 1 & 0 & 0 & 0 \\
110 & 40000 & 12 & 7 & 4 & 1 & 0 & 0 \\
110 & 50000 & 4 & 3 & 1 & 0 & 0 & 0 \\
220 & 120000 & 19 & 17 & 1 & 0 & 0 & 1 \\
220 & 180000 & 4 & 4 & 0 & 0 & 0 & 0 \\
\hline
\end{tabular}

(E: Excellent, B: Better, N: Normal, W: Worse, F: Fault.) 
In order to test the diagnosis' accuracy of the system, some examples in [2] are tested again, and the results are excellent agreement with transformers' actual faults.

\section{Conclusions}

In this paper, we presented some general guidelines of developing an intelligent transformer condition assessment system to help electric utilities optimize the maintenance activities. This proposed framework is open and flexible, so the objective system is easy to be maintained and further developed. A data warehouse has been used to integrate all kind of transformer condition parameters. OAA is employed to compose the multi-agent system that is the main part of the proposed system. Seven application agents are designed to evaluate transformers' conditions synthetically. The initial filed test results got from tests of some transformers based on a prototype system developed by the authors have proven that the framework system is able to produce accurate condition assessment results and is promising for further implementation. Moreover, the maintenance and further development of the objective system is feasible since the present framework is open and flexible.

\section{References}

1. Jie Cheng and Russell Greiner: Comparing bayesian network classifiers. In Proceedings of the 15th Conference on Uncertainty in Artificial Intelligence (UAI'99), Morgan Kaufmann Publishers (1999) 101--107.

2. Mang-Hui Wang: A Novel Extension Method for Transformer Fault Diagnosis, IEEE Transactions on Power Delivery, 18(1) (2003) 164-169 